International Journal of Pure and Applied Mathematics

Volume 102 No. 3 2015, 579-592

ISSN: 1311-8080 (printed version); ISSN: 1314-3395 (on-line version)

url: http://www.ijpam.eu

doi: http://dx.doi.org/10.12732/ijpam.v102i3.14

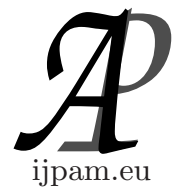

\title{
LOW COST ELLIPTIC CURVE SCALAR MULTIPLICATION FOR CONSTRAINED DEVICES
}

\author{
Jayaprakash Kar \\ Information Security Research Group \\ Department of Information Systems \\ Faculty of Computing \& Information Technology \\ King Abdulaziz University \\ KINGDOM OF SAUDI ARABIA
}

\begin{abstract}
This article proposes the technique to reduce the computation cost of scalar multiplication (SM) on elliptic curve for low processor constraints devices. Also the algorithm using the proposed technique secure against Side Channel Attack. There is no such algorithm is found to solve ECDLP in subexponential time. Equivalent strength of security is provided by ECC with shorter key size as compared to other Public key Cryptosystem. Therefore, ECC has acquired broad attention to research and development especially for low processor devices. Scalar multiplication on ECC operates on point doubling and adding. The secret the key stream consist of bit is ' 0 ' or ' 1 '. While SM operate, doubling of points is followed by addition of points. This is a serious threat for low processor devices. For implementation, it is a challenge to develop algorithms that are not only efficient, but low cost.
\end{abstract}

Key Words: ECDLP, constraint device, scalar multiplication, side channel attack

\section{Introduction}

Due to smaller size in key as compared to the earlier conventional public key cryptography like RSA, Elliptic curve based cryptography (ECC) [1] [6] is having more attractiveness in many cryptographic applications. They are more

Received: April, 42015

(c) 2015 Academic Publications, Ltd. url: www.acadpubl.eu 
compatible for implementation on mobile devices such as Smart Phone, PDA, wireless sensor network etc. Due to the physical characteristics of these low processor devices, consumption of time in power of computations using the secret key can be clearly monitored. Therefore there exists a serious threat known as Side Channel Attack (SCA) against these devices [16]. It is very important to scalar multiplication algorithm on elliptic curve which is not only secure against SCA but also of low cost.

Miller and Koblitz introduced Elliptic curve cryptography (ECC) in 1985,. Security of ECC relies on ECDLP. Attractiveness of ECDLP is there does not exist any sub-exponential algorithms to solve this problem till date [3] [5] [4]. This cryptosystem provides equivalent strength of security as compared to the other conventional cryptosystem with shorter key. Because of this advantage, ECC has gained large interest in both cryptology research community and industry.

But it has been observed that, the attacker analyzes the power traces of cryptographic computations of the SM on Elliptic Curve and differentiates the consumption of power generated by the secret key. The operation SM on elliptic curve contains doubling and addition of points on elliptic curve. During the operation, the key bit is ' 0 ' or ' 1 ' that is doubling of points is followed by addition of points. This is a serious threat for low processor devices. Therefore it is essential to develop an efficient scalar multiplication algorithm on elliptic which is not only SCA-resistant but of low cost.

\section{Introduction to Elliptic Curve}

Let $E$ denotes the elliptic curve on field $F$. Weierstrass equation is given by the curve $\mathcal{E}$ is

$$
\mathcal{E}: v^{2}+\alpha_{1} u v+\alpha_{3} v=u^{3}+\alpha_{2} u^{2}+\alpha_{4} u+\alpha_{6},
$$

where $\alpha_{1}, \alpha_{2}, \alpha_{3}, \alpha_{4}, \alpha_{6} \in K$ and $\Delta \neq 0$.

Let $\Delta$ denotes the discriminant of $\mathcal{E}$.It is given by:

$$
\begin{gathered}
\Delta=-t_{2}^{2} t_{8}-8 t_{4}^{3}-27 t_{6}^{2}+9 t_{2} t_{4} t_{6}, \\
t_{2}=\alpha_{1}^{2}+4 \alpha_{2}, \\
t_{4}=2 \alpha_{4}+\alpha_{1} \alpha_{3}, \\
t_{6}=\alpha_{3}^{2}+4 \alpha_{6},
\end{gathered}
$$




$$
t_{8}=\alpha_{1}^{2} \alpha_{6}+4 \alpha_{2} \alpha_{6}-\alpha_{1} \alpha_{3} \alpha_{4}+\alpha_{2} \alpha_{3}^{2}-\alpha_{4}^{2} .
$$

Let $L$ be the extension of field $F$.

$$
\mathcal{E}(L)=\left\{(u, v) \in L \times L: v^{2}+\alpha_{1} u v+\alpha_{3} v-u^{3}-\alpha_{2} u^{2}-\alpha_{4} u-\alpha_{6}\right\} \cup\{\mathcal{O}\} .
$$

The point $\mathcal{O}$ called the point at infinity.

Consider the following transformation

$$
(u, v) \rightarrow\left(\frac{u-\alpha_{1}^{2}-12 \alpha_{2}}{36}, \frac{v-3 \alpha_{1} u}{216}-\frac{\alpha_{1}^{3}+4 \alpha_{1} \alpha_{2}-12 \alpha_{3}}{24}\right) .
$$

This equation $\mathcal{E}$ can be changed to the curve $v^{2}=u^{3}+\alpha u+\beta$, where $\alpha, \beta \in K$ and $\Delta=-16\left(4 \alpha^{3}+27 \beta^{2}\right)$. Characteristics of $F \neq 2$ or 3 Where $4 \alpha^{3}+27 \beta^{2} \neq 0$ means it does not have multiple roots.

\subsection{Group Law in EC}

Refere to [26] [25], here we described the group laws. Consider the collection of rational points over the elliptic curve form a group with respect to the operation addition. According to secant and tangent's rule, let $(u, v)$ is any arbitrary point lies on the non-super singular curve over binary field. So opposite of the point $u, v)$ is $(u, u+v)$. Consider the field characteristic $>3$. Opposite of the point $(u, v)$ will be $(u,-v)$. For any arbitrary points lies on the elliptic curve over the field of characteristic $>3$, the sum with at infinity is equal to the point itself. Also the sum of opposite point with the point at infinity is the same point.

Consider two arbitrary points $P$ and $Q$ lies on the elliptic curve. Where $Q \neq \pm P . R$ be the point of intersection of the line joining $P$ and $Q$ and the curve. Sum of the point $P$ and $Q$ is equal to opposite of the point $R$. When the two points $P$ and $Q$ are same, we take the tangent at the point of the curve. All the properties of group satisfies on these points. Here we consider the point of infinity is the identity element. in Fig 1 shows the elliptic curve with group laws.

Analytically, let the characteristic of the underlying field $K$ be greater than 3 and let the equation of the elliptic curve $E$ be $v^{2}=u^{3}+\alpha u+\beta$. Let $P$ be any point on the curve, $P+\mathcal{O}=P$. If $P$ has coordinates $\left(u_{1}, v_{1}\right)$ then coordinates of $-P$ are $\left(u_{1},-v_{1}\right)$ and $P+(-P)=\mathcal{O}$.

Let $Q$ be another point on the curve with coordinates $\left(u_{2}, v_{2}\right)$. The sum of $P$ and $Q$ has coordinates $\left(u_{3}, v_{3}\right)$, where

$$
u_{3}=\left(\frac{v_{2}-v_{1}}{u_{2}-u_{1}}\right)^{2}-u_{1}-u_{2}
$$


and

$$
v_{3}=\left(\frac{v_{2}-v_{1}}{u_{2}-u_{1}}\right)\left(u_{1}-u_{3}\right)-v_{1}
$$

If $P=Q$ and $P \neq-P$ then the sum is $2 P$ and it has coordinates $\left(u_{4}, v_{4}\right)$ with

$$
u_{4}=\left(\frac{3 u_{1}^{2}+\alpha}{2 v_{1}}\right)^{2}-2 u_{1}
$$

and

$$
v_{4}=\left(\frac{3 u_{1}^{2}+\alpha}{2 v_{1}}\right)\left(u_{1}-v_{4}\right)-v_{1}
$$

Let the $E$ be a non-super-singular elliptic curve over a binary field $K=F_{2^{n}}$ with equation $v^{2}+u v=u^{3}+\alpha u^{2}+\beta$. On the group $E(K)$, again the point at infinity $\mathcal{O}$ plays the role of identity. The inverse of the point $P\left(u_{1}, v_{1}\right)$ is now $-P=\left(u_{1}, u_{1}+v_{1}\right)$. Let $Q\left(u_{2}, v_{2}\right)$ be another point on the curve with $Q \neq \pm P$. Then $P+Q$ is the point $\left(u_{3}, v_{3}\right)$, where

$$
u_{3}=\lambda^{2}+\lambda+u_{1}+u_{2}+\alpha
$$

and

$$
v_{3}=\lambda\left(u_{1}+u_{3}\right)+u_{3}+v_{1}
$$

with $\lambda=\frac{v_{1}+v_{2}}{u_{1}+u_{2}}$.

The double of the point $P$ is $\left(u_{4}, v_{4}\right)$ where,

$$
v_{4}=\lambda^{2}+\lambda+\alpha
$$

and

$$
v_{4}=u_{1}^{2}+\lambda u_{4}+u_{4}
$$

with $\lambda=u_{1}+v_{1} / u_{1}$.

\subsection{Computation of Addition and Doubling of Points}

Equation of the curve in the simplified is given by

$$
E: v^{2}=u^{3}+\alpha u+\beta
$$

This form is known as Weierstress equation. Where the coefficient $\alpha$ denotes the arbitrary element of the field. But for efficient implementation of ECDBL, [NIST, ANSI, SEC2] recommends the specification of curves taking the coefficient $\alpha=-3$ in the above equation. 

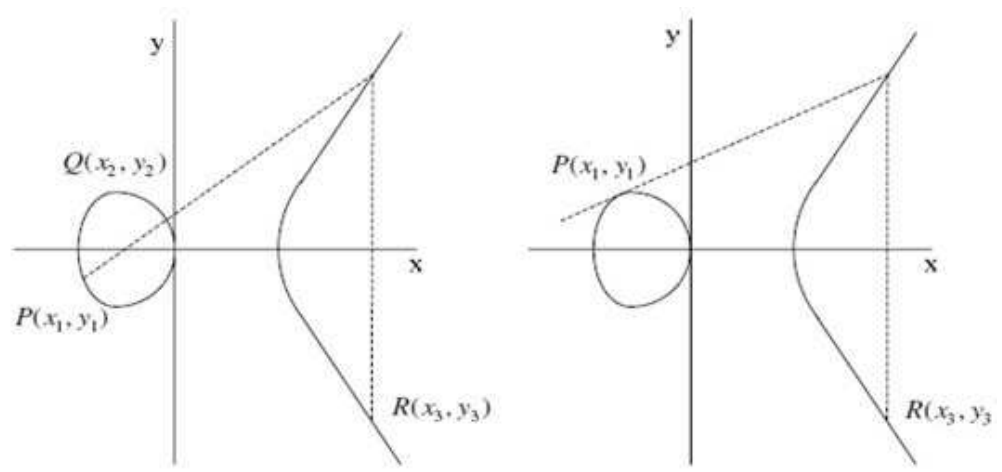

Figure 1: Addition and Doubling of points on Elliptic Curve

\section{Algorithms for Scalar Multiplication}

The most costly operation in ECC is Scalar Multiplication. From implementation point of view, we need to develop low cost algorithms with regards to its computational time and space. We can defined scalar multiplication as $d \times P=P+\cdots(d$ times $) \cdots+P$, where $P$ be any point on $E$ and $d$ is an positive integer. The computation is known as the scalar multiplication operation [12]. Two version of Binary algorithm are defined below to compute the Scalar Multiplication. Where $A D D$ and $D B L$ denote two function addition and doubling of points respectively. The function $A D D$ computes $P+Q$ by talking as input two points $P$ and $Q$. Similarly $D B L$ returns $2 P$ computes its double $2 P$ taking as input $P$.

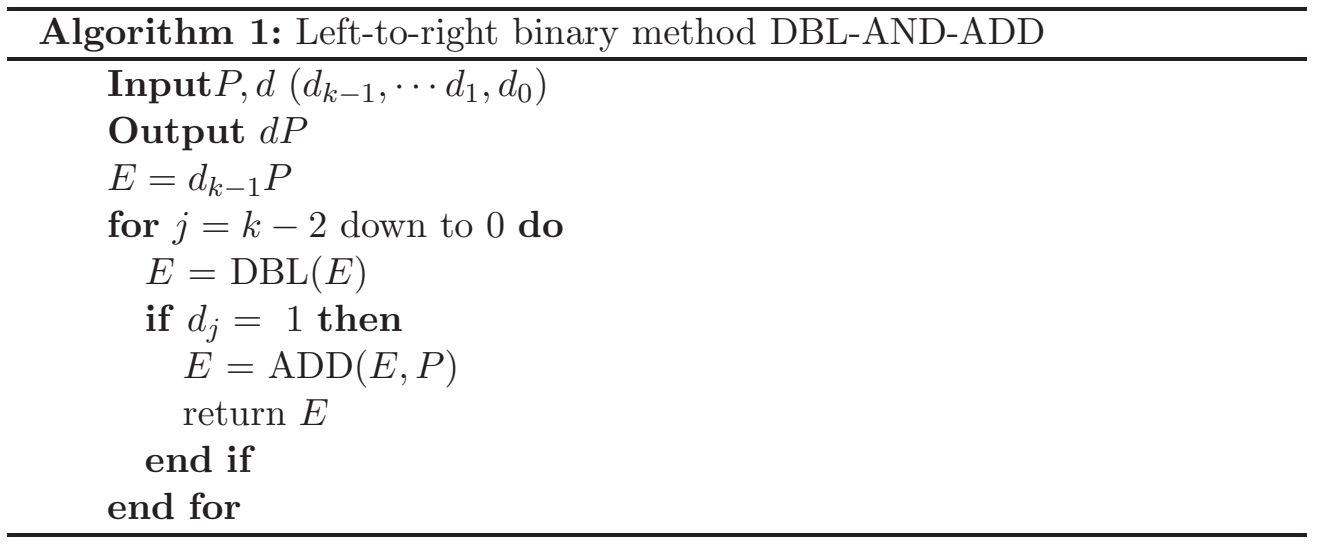




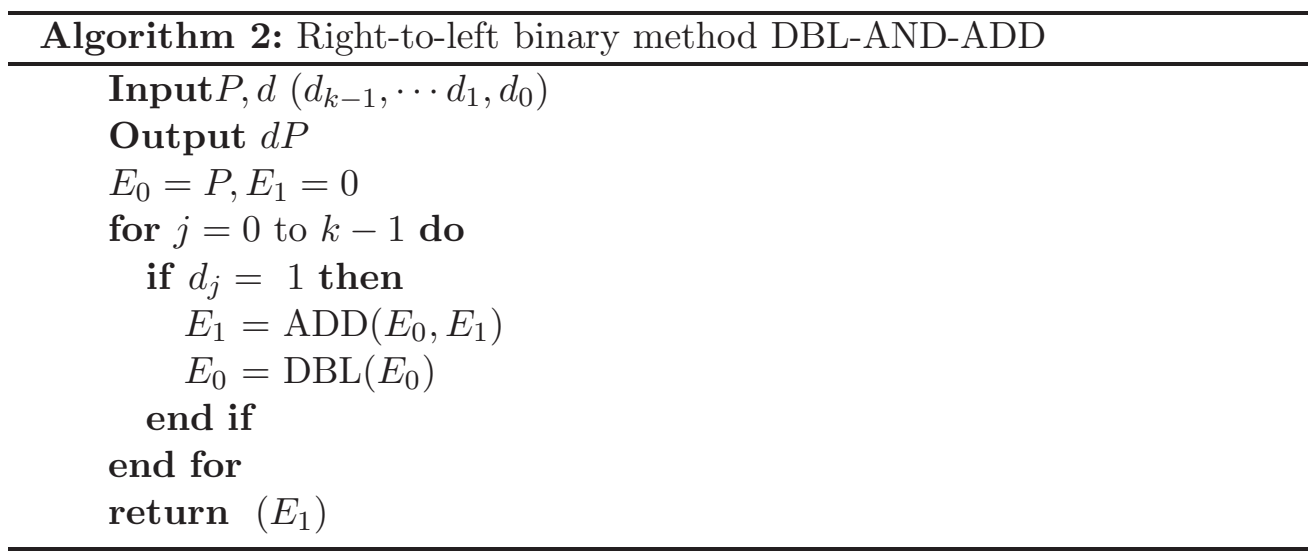

\subsection{Algorithms for Non-Adjacent Form(NAF) and $w$-NAF}

Hamming weight of the multiplier in the Scalar multiplication has the important role to reduce the computation cost. It has been noted the the algorithm is more efficient if the hamming weight less [11] [19] [5]. It is observed that, there is no cost for negation of a point for curve over prime fields, it can be considered, both the addition and subtraction of points are almost same i.e the cost of $(P+Q)$ and $(P-Q)$ are same. Therefore the multiplier can be represented in sighed binary with smaller hamming weight. One of the algorithm to represent signed binary multiplier is Non-Adjacent Form (NAF) [17] [8] [15].

Definition 1. Let binary representation of the multiplier $d$ is $\Sigma_{i} d_{i} 2^{i}$. It can be represented in $\mathrm{NAF} \Longleftrightarrow d_{i} d_{i+1}=0 \forall i$.

Definition 2. Let the width $w$ be a positive integer. The multiplier $d$ is represented by $w$-NAF as $\Sigma_{j=0}^{l-1} d_{j} 2^{i}$, where $d_{j} \in\left[-2^{w-1}, 2^{w-1}-1\right], d$ are odd integers and $d_{j} \neq 0$.

To calculate pre-computation, it needs 1 doubling and $2^{w-2}-1$ additions. On average the no of addition and doubling are $n /(w+1)$ and $n$ respectively.

\section{Proposed Low Cost Scalar Multiplication Algorithm}

Consider the 1's compliment of the binary number, this can be computed by the following equation

$$
n=2^{d}-c_{1}-1
$$



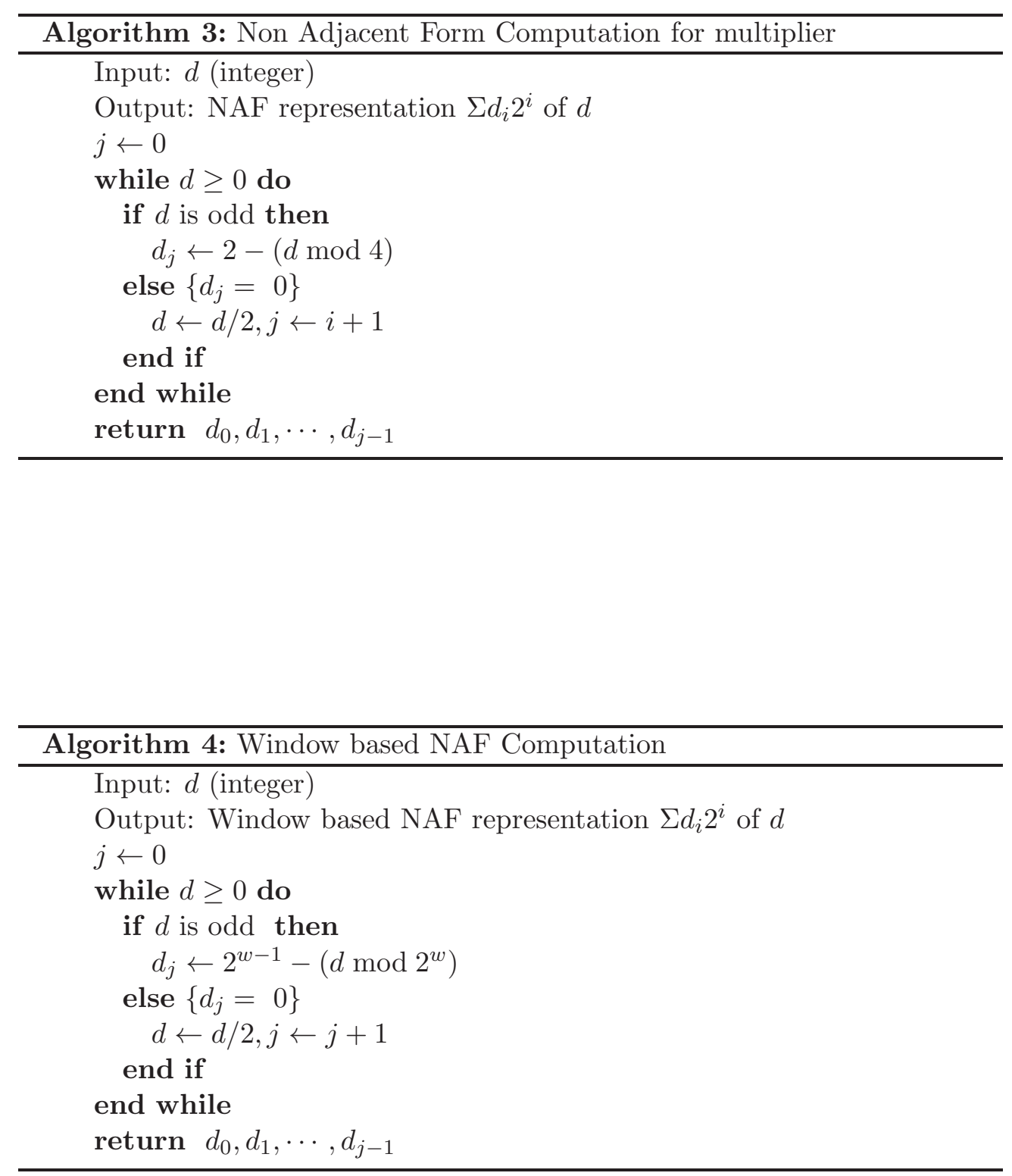

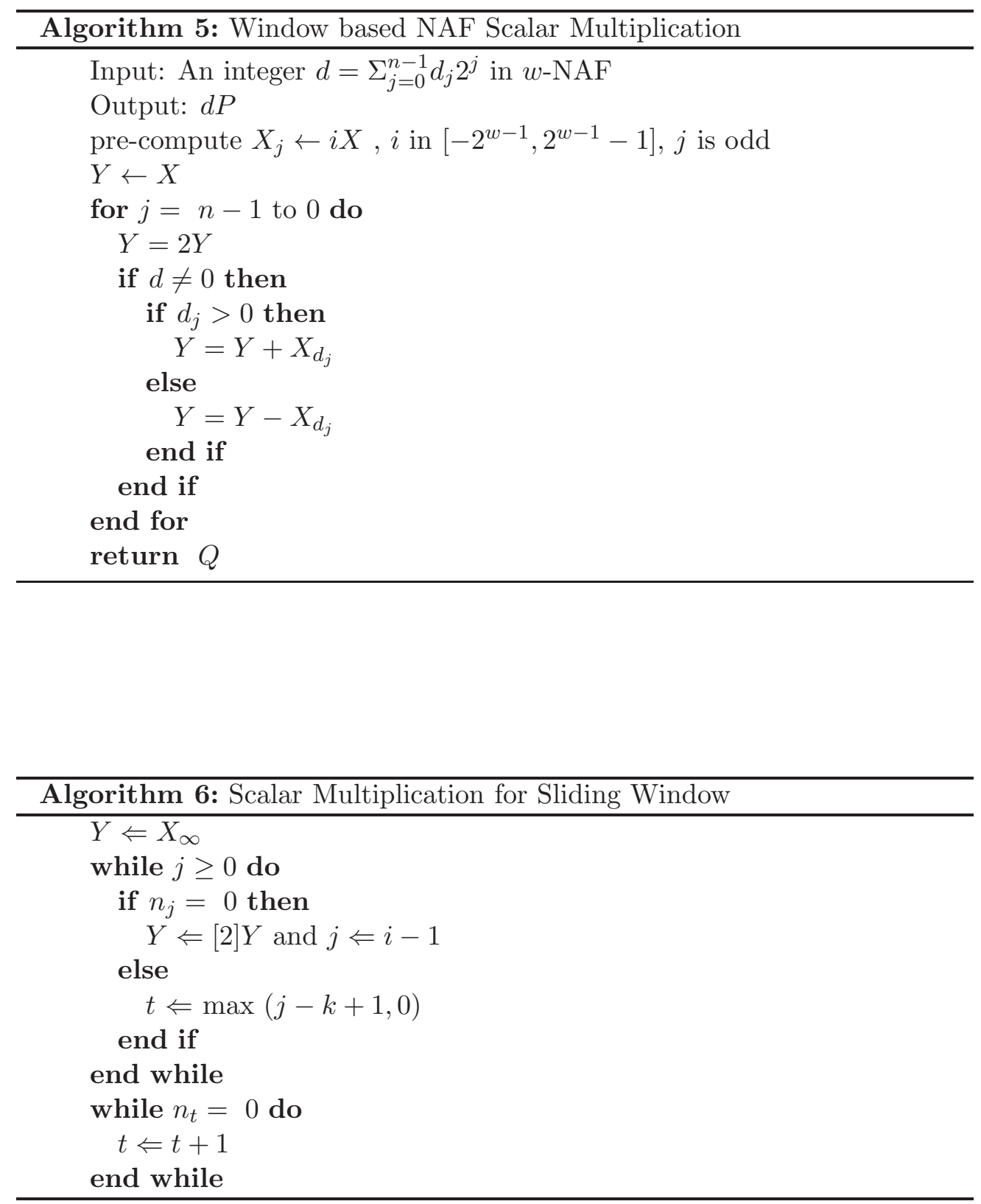


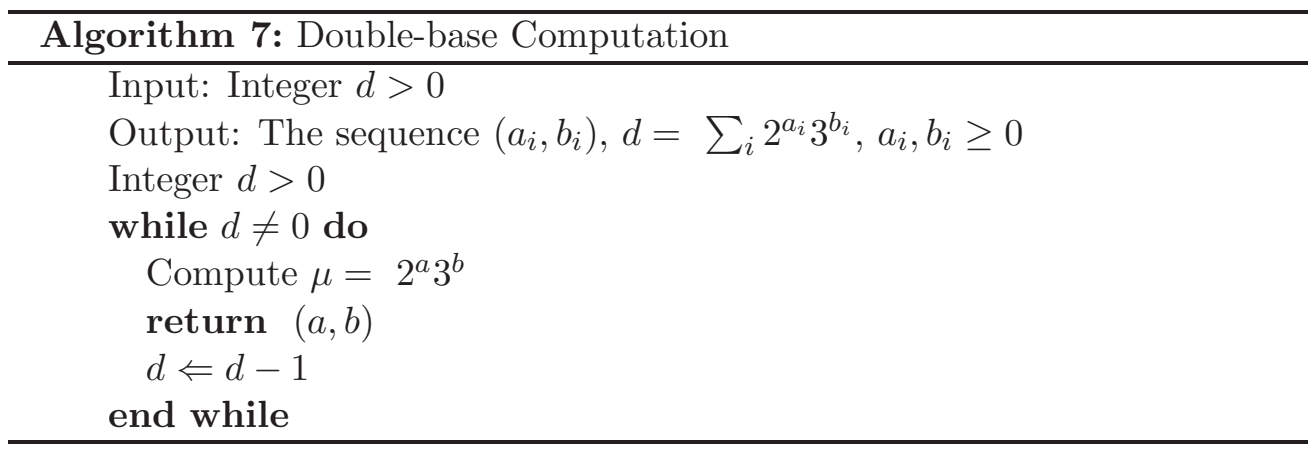

Where $d, c_{1}$ represents number of bits in the binary number $n$ and 1-compliment respectively. If we examine the above equation, the 1 's compliment of any positive integer contains less number of bits that are non-zeros reduces the Hamming weight up to 50 percent. In positive integer scalar the minimal number of zero bits reduces the number of cryptographic operations inversions, multiplication, squaring, addition etc [2]BT06BT07. This reduces the computational cost of the algorithm in Elliptic Curve Cryptography. Consider the following example

Example 1. Let $d=2010$. Binary expansion of this number is (1111101 1010) 2 . 1's compliment $c_{1}=00000100101, d=11$. We can express the number by the above equation as $1642=2^{11}-00000100101-1$. This can be written as $2010=10000000000-00000100101-1$.

The Hamming weight is reduced from 8 to 5 . This save 3 elliptic curve addition. To compute one addition operation, it needs 1 Inverse, 3 Squaring and 6 Multiplication operations. Hence it can be saved total 3 Inverse, 6 Squaring and 6 Multiplication operations.

\subsection{Experiments using Hamming Weight}

This section discusses experiments on the above defined algorithms considering Hamming weights. Let denotes doubling, addition and tripling as $D B L, A D D$ and TRP respectively.

- Double base chains: Consider this algorithm to compute $n P$ given the point $P$ on Elliptic Curve. Let $n=314159$. So $314159 P$ is given by $2(2(2(2(2(2(2(2(2(2(2(2(2(2(2(2(2(2(P))+P))-P)))+P)+P))-P)))+$ $P)+P()))-P$. Starting from the point $P$ results a of $18 D B L$ and $8 A D D$ of the point $P$. We consider the subtraction as addition and count. 
- Double base-2 and -3: The point $314159 P$ can be represented in base 2 and 3 as

$$
\begin{aligned}
& 314159 P=2{ }^{15} 3^{2} P+2^{11} 3^{2} P+2{ }^{8} 3^{1} P+2{ }^{4} 3^{1} P-2{ }^{0} 3^{0} P \\
& =3(2(2(2(2(2(2(2(2(3(2(2(2(2(2(2(2(P))))+P))))+P))))+P)))))-P .
\end{aligned}
$$

This results a lower computation cost in the computation of $314159 P$. Let we start from $P$ get a chin of $2 T R P, 15 D B L$ and $4 A D D$. Consider $1 T R P=1 D B L+1 A D D$. Hence this chain results $17 D B L+6 A D D$ which is having low cost as compared to Double base-2 expansion.

- Sliding-windows with base-2 Consider the Sliding windows base-2 equation for $314159 P$. The expression is

$$
\begin{aligned}
& 2^{16} 5 P-2^{11} 7 P+2^{8} 3 P+2^{4} 3 P-2^{0} P \\
& \quad=2(2(2(2(2(2(2(2(2(2(2(2(2(2(2(2(5 P))))))-7 P)))+3 P))))-P .
\end{aligned}
$$

Let it starts from $P$ result a chain of $17 D B L$ and $7 A D D$.

- Sliding-windows with double-base-2 and-3 The expression of $314159 P$ is given by

$$
\begin{aligned}
& 314159 P=2^{12} 3^{3} 3 P-2^{7} 3^{3} 5 P-2^{4} 3^{1} 7 P-2^{0} 3^{0} P \\
& \quad=3(2(2(2(2(3(3(2(2(2(2(2(2(2(3 P)))))-5 P)))))-7 P)))))-P .
\end{aligned}
$$

Starting from $P$, it counts $3 T P R, 13 D B L$ and $6 A D D$ i.e Total no is $16 D B L+9 A D D$.

Following table summarizes the computational cost as We can observed that, if we can take the hamming weight for 314159 described in section-6 in these above algorithms, this save the number of addition of the scalar multiplication on Elliptic curve and result lowest cost in total computation.

\section{Resistant Against Zero-Value Attacks}

In this article, we have briefed the conventional algorithms for elliptic curve scalar multiplication. Regularity is important for thwart SPA attacks that distinguishes scalar bits from various pattern of operations in a leakage trace. However, regularity may not be adequate to make secure some refine SPA attacks called zero-value attacks. 


\begin{tabular}{|l|l|l|l|l|}
\hline $\begin{array}{l}\text { Type of Algo- } \\
\text { rithm }\end{array}$ & $\begin{array}{l}\text { Double } \\
\text { base chains }\end{array}$ & $\begin{array}{l}\text { Double } \\
\text { base-2 \& 3 }\end{array}$ & $\begin{array}{l}\text { Sliding- } \\
\text { windows } \\
\text { with base-2 }\end{array}$ & $\begin{array}{l}\text { Sliding- } \\
\text { windows } \\
\text { with double- } \\
\text { base-2 \& } \\
3\end{array}$ \\
\hline $\begin{array}{l}\text { Computational } \\
\text { Cost }\end{array}$ & $\begin{array}{l}\text { 18DBL } \\
\text { 8ADD }\end{array}$ & 17DBL+6ADD & 17DBL+ 7ADD & 16DBL+9ADD \\
\hline
\end{tabular}

Table 1: Computation Cost

The point to be consider that makes appear an intermediary point with coordinate of zero-value in computation of $[k] P$ have been described in [20]. From the leakage trace, the appearance of co-ordinate with zero value that allows an SPA attacker to distinguish the value of $\mathrm{k}$ can be traced out and computed. [21] has described this attack elaborately and exploited occurrence of zero-value co-ordinate inside the computations of point addition. This type of attack can be controlled to thwart to randomize the scalar and include dummy operation inside the algorithm [22] [23]. Courr'ege et al. in [27] introduced recently another type of zero-value SPA attacks. In this type of attack, modular exponentiation is targeted. It transposes directly to an attack against specific scalar multiplication algorithms. In fact, SPA adversary can distinguish, whenever multiplication is performed by zero or a digit with low hamming weight value by hardware multiplier of the target device. Generally a hardware multiplier computes the multiplication over the $\mathbb{F}_{q}$. The multiplication comprises $(n \times m)$-bits multiplications. The adversary can get the ideas if the operand of some multiplication over $\mathbb{F}_{q}$ consisting of $n$-bit (resp. $m$-bit) digit to zero or digit with low value hamming weight. This exploits the secret information.

\section{Conclusion}

In this paper, we have proposed the technique to decrease the computation cost of scalar multiplication by considering the hamming weight of the multiplier in Double base chains, Double base-2 and -3, Sliding-windows base-2 and Slidingwindows double-base-2 and-3. It is low cost and resistant on SCA and zero-value attacks. This can be implemented on low processor constrained devices such as smart phone, PDA, wireless sensor, smart card etc [9] [24]. 


\section{References}

[1] N. Koblitz, Elliptic Curves in Cryptography, In Journal of Cryptology.

[2] E. Brier and M. Joye, Weierstrass Elliptic Curves and Side-Channel Attacks

[3] B. Moller, Securing Elliptic curve point against side channel attack

[4] A. Menezes,P.C. van Oorschot, and S.A. Vanstone., Hand book of applied cryptography,C.R.C press, 1997

[5] D. Henkerson, A. Menezes, S. Vanstone, Guide to Elliptic Curve Cryptography, Springer-Verlag, 2004.

[6] W. Diffie and M. Hellman., New direction in cryptography,IEEE Transaction on Information Theory, Vol 22,1976

[7] N. Koblitz., A course in Number Theory and Cryptography, 2nd edition Springer-Verlag-1994

[8] C. Kocher, J. Jaffe and B. June, Differential Power Analysis,CRYPTO99

[9] J. Kar, Authenticated Multiple-Key Establishment Protocol for Wireless Sensor Networks, "Case Studies in Secure Computing Achievements and Trends", CRC Press, Taylor and Francis (New York), Chapter-04, Pages 67-88, Catalogue No- K21540, 2014

[10] E. Brier and M.Joye, Weierstrass elliptic curves and side channel attacks,Springer-2002

[11] K. Koc, David Naccache, and Christof Paar, Cryptograhic Hardware and Embbeded Systems,Springer -Verlag-2001

[12] D. R. Stinson, Cryptograhic :theory and practice,CRC Press, Ibc, 1995

[13] Rana Barua and Tanja Lange, Progress in CryptologyINDOCRYPT 2006, 7th International Conference on Cryptology in India, Kolkata, India, December 1113, 2006, Proceedings, volume 4329 of Lecture Notes in Computer Science, Berlin, 2006. Springer.

[14] Daniel J. Bernstein and Tanja Lange, Explicit-formulas database. http://www. hyperelliptic.org/EFD 
[15] Ian F. Blake, Gadiel Seroussi, and Nigel P. Smart, Elliptic curves in cryptography, volume 265 of London Mathematical Society Lecture Note Series. Cambridge University Press, Cambridge, 1999.

[16] Serdar Boztas and Hsiao-Feng Lu, AAECC 2007, volume 4851 of Lecture Notes in Computer Science, Berlin, 2007. Springer.

[17] K. Okeya, K. Miyazaki and K. Sakuari, A first scalar method with randomized projective coordinates on a Montgomery form Elliptic curve secure against side channel attacks

[18] V. S. Miller, Use of Elliptic Curves in Cryptography.Advances in Cryptology CRYPT' 85

[19] H. Cohen, A. Miyaji, and T. Ono., Efficient Elliptic Curve Exponentiation Using Mixed Co-ordinates,, In Asiacrypt'98, LNCS 1514, pp. 51-65, Springer-Verlag, 1998.

[20] Louis Goubin., A Refined Power-Analysis Attack on Elliptic Curve Cryptosystems. In Yvo Desmedt, editor, Public Key Cryptography, 6th International Workshop - PKC 2003, volume 2567 of Lecture Notes in Computer Science, pages 199-210. Springer, 2003.

[21] Toru Akishita and Tsuyoshi Takagi., Zero-Value Point Attacks on Elliptic Curve Cryptosystem. In Colin Boyd and Wenbo Mao, editors, Information Security, 6th International Conference - ISC 2003, volume 2851 of Lecture Notes in Computer Science, pages 218-233. Springer, 2003.

[22] Jean-Sebastien Coron., Resistance against Differential Power Analysis for Elliptic Curve Cryptosystems. In C etin Kaya Koc and Christof Paar, editors, Cryptographic Hardware and Embedded Systems, First International Workshop - CHES'99, volume 1717 of Lecture Notes in Computer Science, pages 292- 302. Springer, 1999

[23] Mathieu Ciet and Marc Joye., (Virtually) Free Randomization Techniques for Elliptic Curve Cryptography. In Sihan Qing, Dieter Gollmann, and Jianying Zhou, editors, Information and Communications Security, 5th International Conference - ICICS 2003, volume 2836 of Lecture Notes in Computer Science, pages 348-359. Springer, 2003.

[24] J.Kar, A Novel Construction of Aggregate Signcryption Scheme for Smart Card Proceedings of the IEEE 2013 International Conference on CyberEnabled Distributed Computing and Knowledge Discovery, pp 6-13, 2013. 
[25] J.Kar, Provably Secure Signcryption Scheme Based on q-Diffie-Hellman Problem for Wireless Security, Proceedings of the IEEE 2013 International Conference on Cyber-Enabled Distributed Computing and Knowledge Discovery, pp 31-37, 2013.

[26] J.Kar, A Novel Construction of Certificateless Signcryption Scheme for Smart Card Studies in Secure Computing Achievements and Trends, CRC Press, Taylor \& Francis, Book Chapter -22, Pages 437-456, 2014.

[27] Jean-Christophe Courr'ege, Benoit Feix, and Myl'ene Roussellet., Simple power analysis on exponentiation revisited. In Dieter Gollmann, Jean-Louis Lanet, and Julien Iguchi-Cartigny, editors, Smart Card Research and Advanced Application, 9th International Conference - CARDIS 2010, volume 6035 of Lecture Notes in Computer Science, pages 65-79. Springer, 2010. 